\title{
Disseminated Intracranial Tuberculoma Mimicking Neurocysticercosis
}

\author{
Lu Zhengqi, Zhang BingJun, Qiu Wei and Hu Xueqiang
}

\begin{abstract}
Intracranial tuberculoma and neurocysticercosis (NCC) are the most frequent granulomatous infections in the central nervous system. Here we report a 41-year-old man with disseminated intracranial tuberculoma mimicking NCC. The patient complained of relapsing vertigo and vomiting consistent with Bruns syndrome. Serum antibodies against cysticercosis were positive. Magnetic resonance imaging (MRI) of the brain showed multiple disseminated ring-enhanced lesions. An initial diagnosis of NCC was made based on clinical signs and MRI. However, during antiparasitic treatment, the patient exhibited fever, meningitis signs, and positive cerebrospinal fluid findings for tuberculosis. The diagnosis was therefore corrected as tuberculoma. After three months of antituberculous treatment, the patient recovered clinically and on MRI. Our results highlight the importance of differential diagnosis of these two diseases in the early stage.
\end{abstract}

Key words: intracranial tuberculoma, neurocysticercosis, magnetic resonance imaging

(Intern Med 50: 2031-2034, 2011)

(DOI: 10.2169/internalmedicine.50.5518)

\section{Introduction}

Infectious diseases of the central nervous system (CNS), particularly those accompanied by granuloma formation, are worldwide diagnostic challenges, particularly in developing countries. CNS granulomas can be associated with infectious diseases, such as parasitic, bacterial and fungal granulomatous infections (1). Characteristic clinical features of CNS granulomatous infections include seizures, focal neurologic deficits and multiple 'nodular or ring' enhancing lesions on brain magnetic resonance imaging (MRI). The most frequent granulomatous infections in the CNS, with an inflammatory granulomatous response, are intracranial tuberculoma and neurocysticercosis (NCC). However, distinguishing these two diseases during the early stages is difficult. Here we report an interesting case with clinical tuberculous (Bruns syndrome) and disseminated granulomas on MRI mimicking NCC. Our report highlights the importance of the differential diagnosis of these diseases during early stages.

\section{Case Report}

A 41-year-old man presented with 20 days of episodic attacks of vertigo and vomiting triggered by abrupt head movements that lasted a few minutes to one hour. He was asymptomatic between these attacks. He lived in a cysticercosis-endemic area and often used the same kitchen knife and chopping board between raw pork and cooked food. On admission, his brain MRI revealed disseminated hyperintense ring-like lesions with surrounding edema in the hemisphere, cerebellum, brainstem and ventricles on T2weighted images (Fig. 1A). Gadolinium (Gd)-enhanced T1weighted images also showed hyperintense lesions. Biochemical and cytological examinations of cerebrospinal fluid (CSF) were normal (Fig. 2). Colloidal gold immune chromatography assay (Catalog\#43509-020; MP Biomedicals, Singapore) testing of serum and CSF was negative for antituberculous antibodies. Cysticercosis antibodies (measured by enzyme-linked immunosorbent assay) were positive in serum, but negative in CSF. Result of purifiedprotein derivative (PPD) test was negative. His general physical examination and detailed neurological examination was completely 


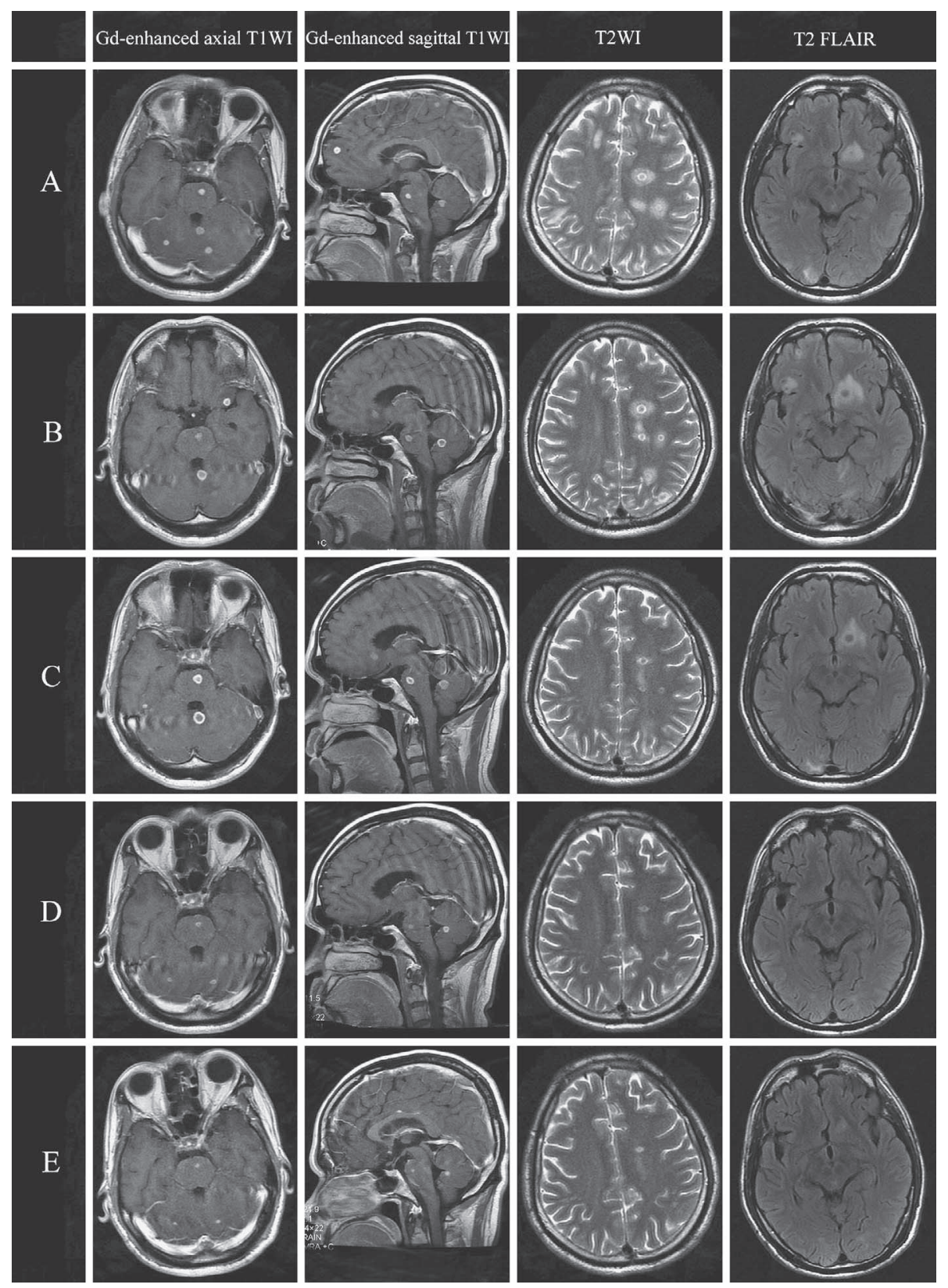

Figure 1. Serial brain MRI findings in the hemisphere, cerebellum, brainstem and ventricle. Gadolinium (Gd)-enhanced T1-weighted images showed nodular hyperintense lesions. Brain MRI revealed hyperintense ring-like lesions along with surrounding oedema on T2-weighted images, and hypointense in T2 fluid attenuated inversion recovery (FLAIR) images. (A) Findings on admission. (B, C) No substantial changes on MRI during antiparasitic medicine. (D, E) Lesions gradually disappeared after antituberculous treatment.

normal. Urinalysis, hemogram, serum biochemical parameters, serum electrolytes, rheumatological factors and tumour markers were also normal. Chest CT revealed no pulmonary lesions. An initial diagnosis of NCC was made and the patient was given albendazole $(20 \mathrm{mg} / \mathrm{kg} / \mathrm{per}$ course/one month per course) and dexamethasone $(0.2 \mathrm{mg} / \mathrm{kg} /$ day $)$. After two courses (two months) of anticysticercosis treatment, repeated brain MRI revealed no substantial lesion changes (Fig. 1B, C). CSF revealed $16 \times 10^{6}$ leucocyte cells/L and normal biochemistry (Fig. 2). During the third course of treatment, the patient developed fever $\left(38.0-39.0^{\circ} \mathrm{C}\right)$. Physical examination showed the meningitis sign of stiff neck and
CSF findings revealed high pressure $\left(>330 \mathrm{mmH}_{2} \mathrm{O}\right)$, proteins of $1.67 \mathrm{~g} / \mathrm{L}$, sugar of $3.83 \mathrm{mmol} / \mathrm{L}$, chloride of 112.1 $\mathrm{mmol} / \mathrm{L}$, and $300 \times 10^{6}$ leucocyte cells/L (75\% lymphocytes) (Fig. 2). Magnetic resonance spectroscopy (MRS) showed choline/creatine ratio $=1.05, \mathrm{~N}$-acetylaspartate/choline ratio= $1.41, \mathrm{~N}$-acetylaspartate/creatine ratio $=1.48$. Therefore, the diagnosis was changed to tuberculoma. Antituberculous treatment (oral isoniazid $300 \mathrm{mg} /$ day, rifampin $450 \mathrm{mg} / \mathrm{day}$, pyrazinamide $1,500 \mathrm{mg} /$ day, ethambutol $750 \mathrm{mg} /$ day, moxifloxacin $400 \mathrm{mg} /$ day, IV dexamethasone $10 \mathrm{mg} /$ day) was administered and three months later, CSF findings were greatly improved. As observed using repeated MRI, the lesions 


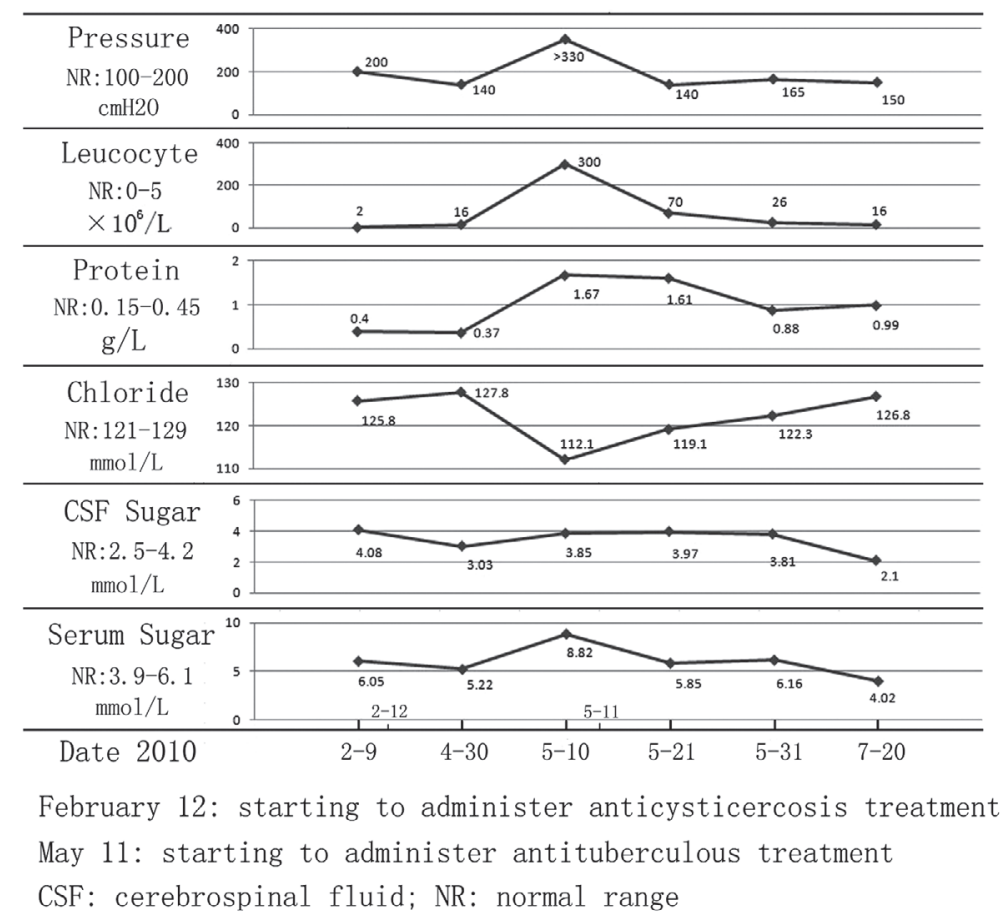

Figure 2. Serial CSF findings and simultaneous blood glucose in the patient.

were disappearing (Fig. 1D, E) and the patient's symptoms were much improved.

\section{Discussion}

Bruns syndrome is characterized by attacks of sudden and severe headache, vomiting and vertigo, triggered by abrupt head movements. To our knowledge, this is the first report on Bruns syndrome caused by intraventricular tuberculoma, with previous reports only in intraventricular tumor and NCC (2-4). The present patient was initially misdiagnosed with NCC because he lived in a cysticercosis-endemic area and had Bruns syndrome without meningitis signs and abnormal CSF findings. Also, serum cysticercosis antibodies were positive and brain MRI showed disseminated granulomas. Multiple tuberculous mycobacterial infections, such as intracranial tuberculoma, are often observed in immunocompromised or in older patients (5). However, the present patient was middle-aged with a normal immune status, with lesions in the lateral and fourth ventricles. Intracranial tuberculoma is typically located in the parenchyma (6) and its occurrence is rarely reported.

Clinical features of intracranial granulomas result from a combination of factors, such as lesion number, stage, localization and the patient's immune response status against granuloma $(7,8)$. Similar clinical features exist in either intracranial tuberculoma or NCC patients. As only $1 \%$ of patients with tuberculosis develop intracranial tuberculoma (9), there are few clinical studies on this condition $(6,10-12)$. Early stage intracranial tuberculoma and NCC are difficult to distinguish and can only be differentiated by clinical and laboratory features and patient history $(10,13)$. For NCC, cysts are hyperintense on $\mathrm{T} 2$ sequences and hypointense on T1 and FLAIR sequences using MRI (7). The scolex is an absolute diagnostic criterion however it is not usually seen on MRI, leading to diagnostic confusion with other infections or intracranial mass lesions. Degenerating cysts appear as contrast-enhancing rings or nodules surrounded by edema. Differential diagnosis of a single degenerating cysticercus from tuberculoma is therefore important, yet difficult.

For tuberculoma, MRI can demonstrate the typical features of contrast-enhancing ring lesions with surrounding oedema (6). We found that Gd-enhanced T1-weighted images showed nodular hyperintense lesions and T2-weighted images showed hyperintense ring-like lesions with surrounding oedema in our patient. Increased use of MRI has shown that infratentorial tuberculomas are more common than previously thought (11). However, neither of these imaging modalities can reliably distinguish tuberculoma from other causes of ring-like lesions, in particular pyogenic bacterial abscess, NCC, toxoplasmosis, or neoplasia. More recently, MRS has shown promise in differentiating these lesions. A large lipid $\mathrm{CH} 2$ peak has been used to specifically identify tuberculomas, which can be distinguished from NCC on the basis of choline/creatine ratio $>1$ in tuberculoma (12). MRS of the present patient showed an increased choline/creatine ratio that was consistent with the report by Pretell et al (12).

In summary, we describe a unique case of intracranial tuberculoma without meningitis signs and positive CSF findings initially mimicking NCC. Our results highlight the importance of distinguishing these two diseases during the early stages. 
The authors state that they have no Conflict of Interest (COI).

Zhengqi Lu and BingJun Zhang contributed equally to this work.

\section{References}

1. daRocha AJ, Maia AC Jr, Ferreira NP, do Amaral LL. Granulomatous diseases of the central nervous system. Top Magn Reson Imaging 16: 155-187, 2005.

2. Krasnianski M, Muller T, Stock K, Zierz S. Bruns syndrome caused by intraventricular tumor. Eur J Med Res 13: 179-181, 2008.

3. Das A, Kesavadas C, Radhakrishnan VV, Nair NS. Teaching NeuroImages: Bruns syndrome caused by intraventricular neurocysticercosis. Neurology 73: e34, 2009.

4. Torres-Corzo J, Rodriguez-della Vecchia R, Rangel-Castilla L. Bruns syndrome caused by intraventricular neurocysticercosis treated using flexible endoscopy. J Neurosurg 104: 746-748, 2006.

5. Udani PM, Parekh UC, Dastur DK. Neurological and related syndromes in CNS tuberculosis. Clinical features and pathogenesis. J Neurol Sci 14: 341-357, 1971.

6. Sonmez G, Ozturk E, Sildiroglu HO, et al. MRI findings of in- tracranial tuberculomas. Clin Imaging 32: 88-92, 2008.

7. Garcia HH, Del Brutto OH. Neurocysticercosis: updated concepts about an old disease. The Lancet Neurology 4: 653-661, 2005.

8. Nicolls DJ, King M, Holland D, Bala J, del Rio C. Intracranial tuberculomas developing while on therapy for pulmonary tuberculosis. Lancet Infect Dis 5: 795-801, 2005.

9. Giese A, Kucinski T, Hagel C, Lohmann F. Intracranial tuberculomas mimicking a malignant disease in an immunocompetent patient. Acta Neurochir (Wien) 145: 513-517; discussion 517, 2003.

10. Bayindir $\mathrm{C}$, Mete $\mathrm{O}$, Bilgic B. Retrospective study of 23 pathologically proven cases of central nervous system tuberculomas. Clin Neurol Neurosurg 108: 353-357, 2006.

11. Wasay M, Kheleani BA, Moolani MK, et al. Brain CT and MRI findings in 100 consecutive patients with intracranial tuberculoma. J Neuroimaging 13: 240-247, 2003.

12. Pretell EJ, Martinot $\mathrm{C} \mathrm{Jr}$, Garcia HH, Alvarado M, Bustos JA, Martinot C. Differential diagnosis between cerebral tuberculosis and neurocysticercosis by magnetic resonance spectroscopy. J Comput Assist Tomogr 29: 112-114, 2005.

13. Del Brutto OH, Rajshekhar V, White AC Jr, et al. Proposed diagnostic criteria for neurocysticercosis. Neurology 57: 177-183, 2001.

(C) 2011 The Japanese Society of Internal Medicine

http://www.naika.or.jp/imindex.html 